\title{
Serious Adverse Events in Pediatric Procedural Sedation Before and After the Implementation of a Pre-Sedation Checklist
}

This article was published in the following Dove Press journal: Journal of Pain Research

\author{
Simona Librov' \\ Itai Shavit ${ }^{2}$ \\ 'Rappaport Faculty of Medicine, \\ Technion-Institute of Technology, Haifa, \\ Israel; ${ }^{2}$ Emergency Department, Ruth \\ Children's Hospital, Rambam Health Care \\ Campus, Haifa, Israel
}

Objective: Procedural sedation is considered by the Joint Commission on Accreditation of Healthcare Organizations as a high-risk procedure when performed in the emergency department (ED). A pre-sedation checklist is a set of items checked before any sedation. We evaluated the impact of a pre-sedation checklist on the rate of serious adverse events (SAE) in a pediatric ED.

Methods: We conducted a retrospective study comparing the rate of SAE in children treated with the combination of ketamine and propofol before and after the implementation of a presedation checklist. The before-and-after periods lasted from 1.1.2013 to 30.6.2016 and from 1.7.2016 to 30.6.2019, respectively. Patient data were extracted from the electronic medical records using an integrated business intelligence information system.

Results: The before-and-after cohorts included 1349 and 1846 patients, respectively. The two groups were similar with regard to age, sex, length and type of procedure, medications dosage, and level of physicians' training. A total of 183/1349 (13.5\%) and 420/1846 (22.7\%) SAE were recorded during the before-checklist and after-checklist periods, respectively $(\mathrm{p}<0.0001)$. The rates of laryngospasm, apnea, and oxygen saturation $\leq 90 \%$ at the beforeand-after checklist periods were $9 / 1349(0.6 \%)$ and 30/1846 (1.6\%); $<<0.05,48 / 1349(3.5 \%)$ and $77 / 1846$ (4.2\%); $\mathrm{p}=0.37$, and $123 / 1349$ (9.1\%) and 312/1846 (16.9\%); $<<0.0001$, respectively. All the SAE were successfully managed by the emergency physicians and no patient required hospitalization due to a SAE.

Conclusion: In this large cohort of ED children who underwent deep sedation, the administration of a pre-sedation checklist was not associated with a reduction in SAE rate.

Keywords: sedation, checklist, adverse events, pediatric

\section{Introduction}

The use of checklists to improve patient care in emergency medicine is increasing. ${ }^{1,2}$ Procedural sedation is a well-established and safe treatment when performed by emergency department (ED) physicians. $^{3-5}$ However, serious adverse events (SAE) such as aspiration, apnea, and laryngospasm might occur, and because of that it is considered by the Joint Commission on Accreditation of Healthcare Organizations as a high-risk procedure. ${ }^{5-7}$ In 2012, a committee of the American College of Emergency Physicians Quality Improvement and Patient Safety Section recommended that preprocedural check and procedure equipment check will be performed before sedation. ${ }^{7}$ Based on international standards for a safe practice of anesthesia, and by using the Revised Pre-anesthetic Set-Up as a conceptual framework, a group of pediatric

\footnotetext{
POB 274, Kibutz Maayan Tzvi, 3080500,

Israel

Tel +972-50-2063239

Fax +972-4-8543109

Email itai@pem-database.org
}

Correspondence: Itai Shavit submit your manuscript | www.dovepress.com
Journal of Pain Research 2020:13 1797-1802

1797 
emergency specialists from Rambam Health Care Campus (RHCC), Haifa, Israel, have created a pre-sedation checklist that meets the unique needs of patients undergoing procedural sedation in the pediatric ED (Figure 1). ${ }^{8,9}$ Since July 2016 the pre-sedation checklist has been incorporated into the department protocols. The objective of the current study was to evaluate the impact of a pre-sedation checklist on the rate of $\mathrm{SAE}$ in a pediatric $\mathrm{ED}$. We hypothesized that by increasing adherence to the sedation protocol, and increasing the safety culture of the teams, the implementation of the checklist would result in a decrease in the rate of SAE.

\section{Methods}

\section{Setting and Study Design}

RHCC is a tertiary trauma care center as well as a referral center for 12 district hospitals. The hospital serves a population of more than two million residents. The pediatric ED has an annual volume of approximately 28,000 patients, and the physicians of the department perform a large number of sedations with the combination of ketamine and propofol, mainly for trauma patients.

We conducted a retrospective study comparing the rate of SAE in children treated with the combination of ketamine and propofol in the ED, before and after the implementation of a pre-sedation checklist. The before-checklist period lasted 3.5 years, from 1 January 2013 to 30 June
2016, and the after-checklist period lasted 3 years, from 1 July 2016 to 30 June 2019. This study was conducted according to the guidelines outlined in the declaration of Helsinki. The patient records and information were anonymized and deidentified before analysis. The Institutional Review Board of Rambam Health Care Campus (No. 0439-19) approved this study without the requirement of obtaining informed consent.

\section{Sedation Protocol}

The combination of ketamine and propofol has gained popularity in recent years, because the drugs are thought to complement one another., ${ }^{6,10}$ The antiemetic and anxiolytic properties of propofol mitigate the vomiting and emergence reactions induced by ketamine, while the sympathomimetic effect of ketamine mitigates the hypotension induced by propofol. ${ }^{11}$ In the pediatric ED of RHCC children are treated with the combination of ketamine and propofol regularly as of 2012. According to ED protocol, if a patient undergoing painful procedure is older than 12 months, has an American Society of Anesthesiologists physical status classification $\leq 2$, and has been fasting for $\geq 2$ hours, $\mathrm{s} /$ he can be managed with deep sedation. Department protocol recommends using propofol and ketamine in separate doses, with ketamine given first to decrease the risk of injection-site pain with propofol. ${ }^{10}$ Ketamine is administered in a loading dose of $1 \mathrm{mg} / \mathrm{kg}$ followed by propofol in a loading dose of $1 \mathrm{mg} / \mathrm{kg}$. Propofol

\section{Pediatric Emergency Department pre-sedation checklist}

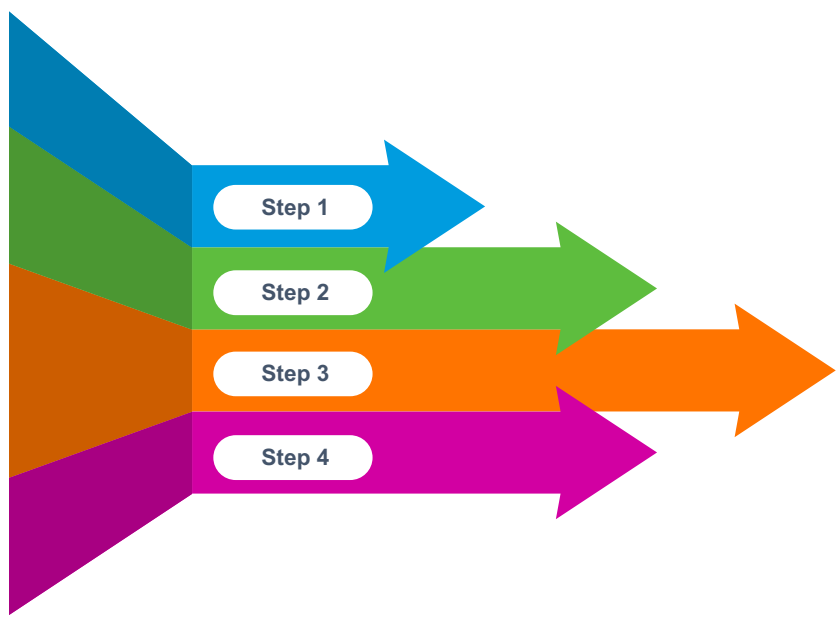

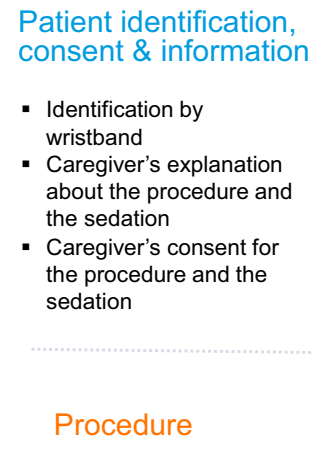

- Equipment and instrumentation present

- Tetanus Toxoid - YES/NO

- Intravenous antibiotics - YES/NO

- Caregiver's consent
Patient risk

assessment

- Absence of chronic disease

- No sedation medication allergy

- Fasting status $\geq 2$ hours

Equipment \& medications check

- Oxygen check

- Bag and mask check

- Suction check

- Monitor check:

ECG/Pulse Oximetry/Capnography

Figure I Pediatric Emergency Department pre-sedation checklist protocol. 
boluses of $1 \mathrm{mg} / \mathrm{kg}$ are then administered every $0.5-2 \mathrm{~min}$ patient reaches a state of deep sedation, as defined by a University of Michigan Sedation Scale score of 3 (deep sleep/arouses to deeper physical stimuli). ${ }^{12}$

\section{Design and Implementation of the Pre- Sedation Checklist}

Results of a landmark paper conducted by the World Health Organization revealed that the introduction of a team-based surgical safety checklist reduced death and complication rates of adult patients undergoing noncardiac surgery. ${ }^{13}$ An important finding of this study was the fact that the checklist leads to changes in the behavior of individual surgical teams. This finding seems to suggest that the checklist may have affected the safety culture of these teams. A study that used a pre-anesthetic checklist revealed that in the intense environment of the operating room, preparatory steps may be missed by the anesthesiologists. ${ }^{9}$ The preanesthetic checklist is a set of items designed for anesthesiologists to check equipment adequately and to minimize missed steps in the preparation for anesthesia. Given the highly stressful work environment in which emergency physicians practice, key preparation steps for procedural sedation may be missed as well. Based on this knowledge, attending pediatric emergency physicians at RHCC have decided to embrace the concept of checklist and to create a pre-sedation checklist, aimed at reducing SAE related to procedural sedation. A panel of three anesthesiologists, expert in pediatric sedation, independently reviewed the pre-sedation checklist and unanimously endorsed the face validity of the instrument for ED procedural sedation. The checklist includes 15 items that seem conducive to safe patient outcome in the ED. The items cluster into four categories: patient identification, consent and information, patient risk assessment, procedure, and equipment and medications check (Figure 1). Pre-sedation recording is done before the sedation when the child and the caregivers are present in the procedures room. Each of the checklist items is scored "yes" or "done". Only when the nurse verifies that all 15 checklist items are met, the sedation and the procedure can begin. Three months before implementing the presedation checklist, the tool was tested by 5 ED nurses. The nurses evaluated 20 procedural sedations in the ED in a blinded fashion, and were satisfied with the instrument's ease of use and the time required for it (5-10 minutes). On 1 July 2016, the pre-sedation checklist had been incorporated into the ED protocols and is being used since then before every sedation.

\section{Data Collection and Selection of Participants}

RHCC patient data management system ("Prometheus", integrated electronic medical records system, Haifa, Israel) is a computerized mandatory working tool for all physicians, nursing staff and any ED healthcare personnel. All patients seen in RHCC have their episode of care recorded in the Prometheus. ${ }^{10}$ The Prometheus includes a sedation recording section that is used for procedural sedation data in the pediatric ED. Data is recorded in real-time by the sedation nurse during the sedation. The sedation section in the Prometheus includes procedure begin time, procedure end time, vital signs, medication administration, medication dose, and any SAE related to the sedation. ${ }^{14}$ Recording of SAE is based on standardized definitions from the Quebec guidelines, a consensus-based document developed by North American experts in ED pediatric procedural sedation, to report sedation data and adverse events requiring intervention. ${ }^{15}$ Based on Prometheus, the Information Technology department of the hospital developed a business intelligence information system (BIINS) that provides reporting and analytical functions across multiple datasets. The BIINS enables automatic extraction of patient data from the electronic medical records according to criteria set by researchers. The following data were extracted from the BIINS for each patient who was sedated with the combination of ketamine and propofol: demographics (age, sex), procedure begin time, procedure end time, medications dosage, sedation physicians' name and level of training, and the following adverse events: aspiration, laryngospasm, apnea, and oxygen saturation $\leq 90 \%$. Any unplanned hospital admission due to sedation was also extracted from the BIINS.

\section{Study Outcome Measures}

The outcome measures for the study were the rate of SAE defined as clinically suspected aspiration (coughing with decreased oxygen saturation), laryngospasm, apnea, and oxygen saturation $\leq 90 \%$, and unplanned hospital admission due to sedation.

\section{Statistical Analysis}

Descriptive statistics were generated, including means and standard deviations (SD) for continuous variables and 
frequencies, medians, and interquartile ranges (IQR) for the categorical variables. Rate of adverse events before and after the implementation of the checklist was compared using the Chi-squared test and the Fisher's Exact test. All statistics were calculated with StatsDirect (version 2.6.6; StatsDirect Limited, Cheshire, UK).

\section{Results}

Overall, 3195 patients with a mean age of $8.2 \pm 4.6$ years were sedated with the combination of ketamine and propofol in the ED during the 6.5 years of the study. Sedation checklist was used before all the procedures during the study period. The before and after cohorts included 1349 and 1846 patients, respectively. The two groups were similar with regard to age, sex, length of procedure, type of procedure, medications dosage, and level of physician training (Table 1). A total of 183/1349 (13.5\%) and 420/1846 (22.7\%) SAE were recorded during the before-checklist and after-checklist periods, respectively $(\mathrm{p}<0.0001)$. No cardiovascular events were recorded. All the procedures were successfully completed and the patients were safely discharged from the ED. All the SAE were successfully managed and no patient required hospitalization due to a SAE. The rates of laryngospasm, apnea, and oxygen saturation $\leq 90 \%$ at the before-and-after checklist periods were (9/ $1349(0.6 \%)$ and 30/1846 (1.6\%)); $\mathrm{p}<0.05 \quad(48 / 1349$ $(3.5 \%)$ and $77 / 1846(4.2 \%)) ; p=0.37$, and $(123 / 1349$ $(9.1 \%)$ and $312 / 1846(16.9 \%)) ; p<0.0001$, respectively. There were 8 episodes of clinically suspected aspiration; 5 at the before-checklist period, and 3 at the after-checklist period $(\mathrm{p}=0.29)$.

\section{Discussion}

In the current study, we tested the impact of a pre-sedation checklist on the incidence of SAE in a large cohort of children who were sedated with the combination of ketamine and propofol in a pediatric ED. The major finding of the study is that the rates of SAE increased after the administration of the checklist. All the patients were safely discharged from the ED after observation. Prior to the study, we assumed that the administration of a pre-sedation checklist would have reduced the number of SAE by improving the adherence to sedation protocols, and by positively affecting the safety culture of the ED team. The results clearly suggest that administration of pre-sedation checklist was not associated with a reduction in $\mathrm{SAE}$ rate. We speculate that the main reason for the surprising rise in SAE is a change in the work environment throughout the years of the study (2013-2019).
It had been shown that ED crowding, and work pressure on the staff can affect the risk of procedures. ${ }^{7}$ In our study, there were 1349 sedations with the combination of ketamine and propofol during the first 3.5 years (before-checklist period), versus 1846 sedations with the combination of ketamine and propofol during the following 3 years (after-checklist period). This significant increase from 385 to 615 sedations per year is the result of an increase in the annual volume of patients in the ED; from 13,000 patients in 2013 to 28,000 patients in 2019. It is important to note that the staff in this department is responsible for a significant percentage of high-acuity patients (including major trauma) and regularly performs sedations in these relatively high-risk patients. ${ }^{16} \mathrm{~A}$ more loaded ED means more work, more tasks and more pressure on the emergency physicians. This environmental change might have a negative impact on the performance of the emergency physician who is working in a stressful environment and is expected to multitask effectively. ${ }^{17}$ During the shift and prior to the sedation, the physician treated other patients and had other tasks that he/she needed to return to once the sedation is completed. We believe that the increase in patient load led to a desire to complete the sedation as early as possible in order to return to the other tasks that were waiting for him/her. Our speculation of patient overload is backed by a survey of open questions we conducted among the 18 physicians of the department. Fourteen physicians mentioned "an urge to finish the sedation as early as possible so that they could see other patients". The sedation protocol allows for propofol boluses every $0.5-2 \mathrm{~min}$ until patient reaches a state of deep sedation. However, some physicians admitted that the time of administering the medications were not always documented accurately, and propofol was administered in intervals of less than 30 seconds.

We found only one study that investigated the impact of a pre-sedation checklist on adverse events related to sedation. The investigators examined the use of a presedation checklist in a mix cohort of 773 children in an Intensive Care Unit, an ED, and in-hospital wards. They did not find a reduction in the rate of SAE. ${ }^{18}$

A pre-sedation checklist has possible advantages that were not tested in our study. The fact that the team responsible for the sedation gather together with the child and his/her parents before the sedation and provided them appropriate explanation about the sedation, likely led to an improved parental satisfaction. It is also reasonable to believe that the pre-sedation checklist positively affected the collaboration between ED physicians, nurses, and surgeons. 
Table I Characteristics of Procedures Performed in Patients Sedated with the Combination of Ketamine and Propofol Before and After the Administration of a Pre-Sedation Checklist

\begin{tabular}{|c|c|c|}
\hline & $\begin{array}{l}\text { Before-Checklist Period } \\
(n=1349)\end{array}$ & $\begin{array}{l}\text { After-Checklist Period } \\
(n=1846)\end{array}$ \\
\hline $\begin{array}{l}\text { Age, mean } \pm S D \\
\text { Males/Females } \\
\text { Length of procedure, min, mean } \pm S D\end{array}$ & $\begin{array}{l}8.0 \pm 4.5 \\
937 / 4 \mid 2 \\
17.2 \pm 11.8\end{array}$ & $\begin{array}{l}8.3 \pm 4.7 \\
1252 / 594 \\
15.8 \pm 10.9\end{array}$ \\
\hline $\begin{array}{l}\text { Type of procedures } \\
\text { Fracture reduction or management of dislocation } \\
\text {-Upper limb, } n(\%) \\
\text {-Lower Limb, } n(\%) \\
\text { Complex or deep laceration repair, } n(\%) \\
\text { Abscess incision and drainage, } n(\%) \\
\text { Soft-tissue foreign-body extraction, } n(\%) \\
\text { Intraoral procedure, } n(\%) \\
\text { Arthrocentesis, } n(\%) \\
\text { Percutaneous CSF sampling of ventriculoperitoneal shunt, } n(\%) \\
\text { Other (eg, nail bed procedure, removal of K-wire, insertion of nasogastric } \\
\text { tube), } n(\%)\end{array}$ & $\begin{array}{l}702(52.1) \\
61(4.6) \\
361(26.7) \\
73(5.4) \\
41(3.0) \\
22(1.6) \\
10(0.8) \\
8(0.6) \\
71(5.2)\end{array}$ & $\begin{array}{l}948(51.3) \\
109(5.9) \\
402(21.9) \\
148(8.0) \\
52(2.9) \\
25(1.3) \\
32(1.7) \\
14(0.7) \\
116(6.3)\end{array}$ \\
\hline $\begin{array}{l}\text { Level of physician training } \\
\text { Attending physicians, } \mathrm{n}(\%) \\
\text { Pediatric residents, } \mathrm{n}(\%)\end{array}$ & $\begin{array}{l}751(55.7) \\
598(44.3)\end{array}$ & $\begin{array}{l}1065(57.7) \\
781(42.3)\end{array}$ \\
\hline $\begin{array}{l}\text { Dosage } \\
\text { Mean total ketamine dose, } \mathrm{mg} / \mathrm{kg} \\
\text { Mean total propofol dose, } \mathrm{mg} / \mathrm{kg}\end{array}$ & $\begin{array}{l}1.04 \\
2.45\end{array}$ & $\begin{array}{l}1.02 \\
2.60\end{array}$ \\
\hline
\end{tabular}

Abbreviations: SD, standard deviation; CSF, cerebrospinal fluid.

This study has several limitations. Firstly, team-related checklists have well-known inherent limitations such as relying on good communication between team members, and cultural challenges, such as hierarchy and perceptions of professionalism. ${ }^{19}$ Secondly, this study has the inherent limitations of a retrospective study design, including dependence on the quality of documentation recording. The information was extracted using a business intelligence information system; therefore, misinterpretation or abstractor bias had no impact. Thirdly, although the pre-sedation checklist has face validity it has not been subjected to psychometric testing to determine reliability and validity. Fourthly, it is theoretically possible, that after checklist administration, recording of SAE by the nurses was more accurate than before checklist administration. We do not think that this is the case because the nurses in this department are well trained in sedation recording as of 2013 and had no incentive for increased recording of SAE after checklist implementation. Furthermore, the after-checklist period lasted three years, a long enough period to eliminate a possible effect of the introduction of a new instrument.
In conclusion, in this large cohort of ED children who underwent deep sedation, the administration of a pre-sedation checklist was not associated with a reduction in SAE rate.

\section{Abbreviations}

ED, emergency department; SAE, serious adverse events; BIINS, business intelligence information system.

\section{Author Contributions}

All authors made substantial contributions to conception and design, acquisition of data, or analysis and interpretation of data; took part in drafting the article or revising it critically for important intellectual content; agreed to submit to the current journal; gave final approval of the version to be published; and agree to be accountable for all aspects of the work.

\section{Funding}

For the 2 authors, there are no potential conflicts of interest, real or perceived in the study design, the collection, 
analysis, and interpretation of data, the writing of the report, and the decision to submit the paper for publication. No honorarium, grant, or other form of payment was given to anyone to produce the manuscript. The authors have no conflict of interest.

\section{Disclosure}

The authors report no conflicts of interest in this work.

\section{References}

1. Hearns S. Checklists in emergency medicine. Emerg Med J. 2018;35 (9):530-531. doi:10.1136/emermed-2018-207782

2. Smith KA, High K, Collins SP, Self WH. A preprocedural checklist improves the safety of emergency department intubation of trauma patients. Acad Emerg Med. 2015;22(8):989-992. doi:10.1111/acem. 12717

3. Pacheco GS, Ferayorni A. Pediatric procedural sedation and analgesia. Emerg Med Clin North Am. 2013;31(3):831-852. doi:10.1016/j. emc.2013.04.002

4. Godwin SA, Burton JH, Gerardo CJ, et al. Clinical policy: procedural sedation and analgesia in the emergency department. Ann Emerg Med. 2014;63(2):247-258. doi:10.1016/j.annemergmed.2013.10.015

5. Bhatt M, Johnson DW, Chan J, et al. Sedation safety study group of pediatric emergency research Canada (PERC). Risk factors for adverse events in emergency department procedural sedation for children. JAMA Pediatr. 2017;171(10):957-964. doi:10.1001/ jamapediatrics.2017.2135

6. Grunwell JR, Travers C, Stormorken AG, et al. Pediatric procedural sedation using the combination of ketamine and propofol outside of the emergency department: a report from the Pediatric Sedation Research Consortium. Pediatr Crit Care Med. 2017;18(8):e356e363. doi:10.1097/PCC.0000000000001246

7. Pines JM, Kelly JJ, Meisl H, et al. Procedural safety in emergency care: a conceptual model and recommendations. Jt Comm J Qual Patient Saf. 2012;38(11):516-526. doi:10.1016/s1553-7250(12)38069-0

8. Merry AF, Cooper JB, Soyannwo O, Wilson IH, Eichhorn JH. International standards for a safe practice of anesthesia 2010. Can J Anaesth. 2010;57(11):1027-1034. doi:10.1007/s12630-010-9381-6
9. Demaria S Jr, Blasius K, Neustein SM. Missed steps in the preanesthetic set-up. Anesth Analg. 2011;113(1):84-88. doi:10.1213/ ANE.0b013e318219645e

10. Scheier E, Gadot C, Leiba R, Shavit I. Sedation with the combination of ketamine and propofol in a pediatric ed: a retrospective case series analysis. Am J Emerg Med. 2015;33(6):815-817. doi:10.1016/j. ajem.2015.03.033

11. Sharieff GQ, Trocinski DR, Kanegaye JT, et al. Ketamine-propofol combination sedation for fracture reduction in the pediatric emergency department. Pediatr Emerg Care. 2007;23(12):881-884. doi:10.1097/pec.0b013e31815c9df6

12. Malviya S, Voepel-Lewis T, Tait AR, et al. Depth of sedation in children undergoing computed tomography: validity and reliability of the University of Michigan Sedation Scale (UMSS). $\mathrm{Br} J$ Anaesth. 2002;31(2):241-245. doi:10.1093/bja/88.2.241

13. Haynes AB, Weiser TG, Berry WR, et al. Safe Surgery Saves Lives Study Group. A surgical safety checklist to reduce morbidity and mortality in a global population. $N$ Engl J Med. 2009;360(5):491949. doi:10.1056/NEJMsa0810119

14. Samuel N, Shavit I, Steiner IP, Kimia AA. A proactive approach to ED information technology: the case of pediatric procedural sedation. Am J Emerg Med. 2016;34(9):1901-1902. doi:10.1016/j.ajem.2016. 06.102

15. Bhatt M, Kennedy RM, Osmond MH, et al. Consensus-based recommendations for standardizing terminology and reporting adverse events for emergency department procedural sedation and analgesia in children. Ann Emerg Med. 2009;53(4):426-435. doi:10.1016/j. annemergmed.2008.09.030

16. N BA, Samuel N, Ben-Lulu H, Bahouth H, Shavit I. Procedural sedation in non-intubated children with severe trauma - a single center study. Am J Surg. 2019;218(1):95-99. doi:10.1016/j. amjsurg.2018.08.016

17. Skaugset LM, Farrell S, Carney M, et al. Can you multitask? Evidence and limitations of task switching and multitasking in emergency medicine. Ann Emerg Med. 2016;68(2):189-195. doi:10.1016/ j.annemergmed.2015.10.003

18. Kahlenberg L, Harsey L, Patterson M, et al. Implementation of a modified WHO pediatric procedural sedation safety checklist and its impact on risk reduction. Hosp Pediatr. 2017;7(4):225-231. doi:10. 1542/hpeds.2016-0089

19. Catchpole K, Russ S. The problem with checklists. BMJ Qual Saf. 2015;24(9):545-549. doi:10.1136/bmjqs-2015-004431
Journal of Pain Research

\section{Publish your work in this journal}

The Journal of Pain Research is an international, peer reviewed, open access, online journal that welcomes laboratory and clinical findings in the fields of pain research and the prevention and management of pain. Original research, reviews, symposium reports, hypothesis formation and commentaries are all considered for publication. The manuscript management system is completely online and includes a very quick and fair peer-review system, which is all easy to use. Visit http:// www.dovepress.com/testimonials.php to read real quotes from published authors. 